\title{
Kyste parodontal latéral
}

\section{RÉSUMÉ}

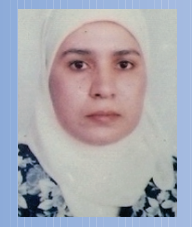

Saliha CHBICHEB

Professeur assistant

en odontologie chirurgicale,

Faculté de médecine dentaire, BP 6212,

Rabat-Instituts, Maroc.

Service d'odontologie chirurgicale,

C.C.T.D. - C.H. Ibn Sina - Rabat, Maroc.

Abdelkader BENNANI

Résident en odontologie chirurgicale.

Service d'odontologie chirurgicale,

C.C.T.D. - C.H. Ibn Sina - Rabat, Maroc.

\section{Bouchra TALEB}

Professeur agrégé

en odontologie chirurgicale.

Service d'odontologie chirurgicale,

C.C.T.D. - C.H. Ibn Sina - Rabat, Maroc.

\section{Wafaa EL WADY}

Professeur de l'Enseignement supérieur, Chef de service d'odontologie chirurgicale. Service d'odontologie chirurgicale,

C.C.T.D. - C.H. Ibn Sina - Rabat, Maroc.

Le kyste parodontal latéral est classé parmi les kystes odontogènes des maxillaires (OMS 1992).

Les auteurs rapportent le cas d'un kyste parodontal latéral chez une femme de race blanche âgée de 33 ans. Cliniquement, il se manifestait par une tuméfaction siégeant au niveau de la région maxillaire antérieure. Radiologiquement, il se présentait par une image radioclaire siégeant entre les racines de la 12 et la 13 . Après un traitement chirurgical conservateur, aucun signe de récidive n'a été rapporté.

Le kyste parodontal latéral se manifeste par des signes cliniques, radiologiques et histologiques non spécifiques et pose des problèmes de diagnostic différentiel avec les kystes et les tumeurs odontogènes bénignes des maxillaires.

kyste parodontal latéral

kyste gingival de l'adulte

- kystes odontogènes 


\section{Introduction}

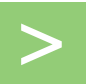

Le kyste parodontal latéral est un kyste épithélial odontogène lié au développement. Cette lésion kystique est souvent asymptomatique et de découverte radiolo-

\section{Observation}

Mme M.S., âgée de 33 ans, a consulté pour une tuméfaction indolore, d'évolution lente et siégeant au niveau de la région antérieure maxillaire droite.

L'examen endobuccal a révélé la présence d'une voussure vestibulaire, recouverte d'une muqueuse d'aspect normal et s'étendant dans le vestibule buccal de la 12 à la 13 (fig. 1). À la palpation, la tuméfaction a été indolore, faisant corps avec l'os.

L'examen dentaire a montré la présence de malpositions au niveau de la région incisivocanine maxillaire droite. Les dents proximales à la tuméfaction étaient saines et répondaient positivement aux tests de vitalité pulpaire.

Le diagnostic clinique a été en faveur d'un kyste ou d'une tumeur bénigne.

L'examen radiographique (fig. $\mathbf{2}$ et $\mathbf{3}$ ) a montré la présence d'une image radioclaire bien limitée, de gique fortuite. Les auteurs rapportent le cas d'un kyste parodontal latéral en présentant ses caractéristiques cliniques, radiologiques et anatomopathologiques.

forme ovalaire et qui siégeait entre les racines de la 12 et de la 13. L'image présentait une double tonalité, ce qui laisse prévoir l'effacement complet d'une corticale et partiel de l'autre.

À partir de ces renseignements cliniques et radiologiques plusieurs diagnostics peuvent être évoqués: kyste parodontal latéral, kyste globulomaxillaire ou tumeur odontogène bénigne.

Le traitement a consisté en l'énucléation de la lésion (fig. 4, 5, 6). La pièce opératoire qui mesure $2 \times 0,5 \times 0,2 \mathrm{~cm}$ a été envoyée pour un examen anatomopathologique (fig. 7). Ce dernier a montré la présence d'un épithélium fin présentant des invaginations tapissant un chorion non inflammatoire. Cet aspect histologique est en faveur du kyste parodontal latéral (fig. 8). Une surveillance régulière postopératoire a été effectuée et a consisté en des contrôles cliniques et radiologiques réguliers.

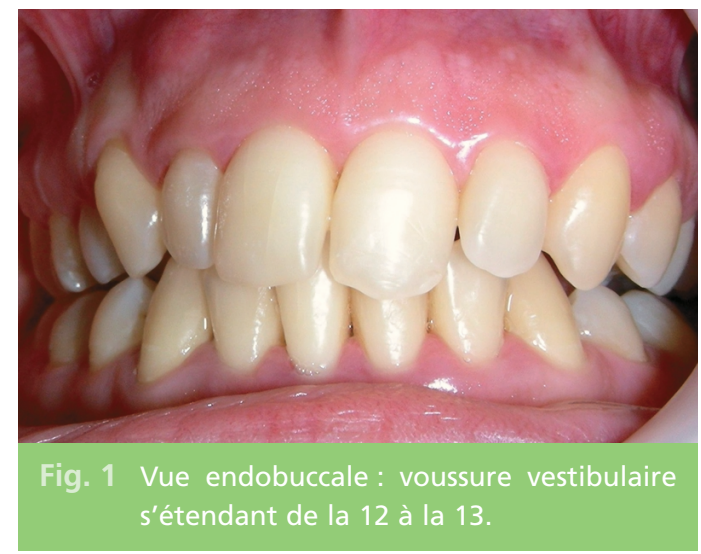




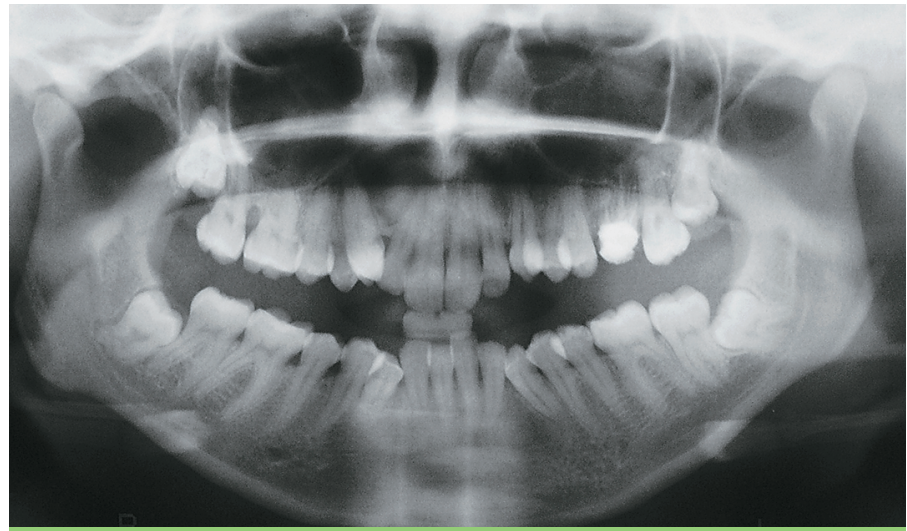

Fig. 2

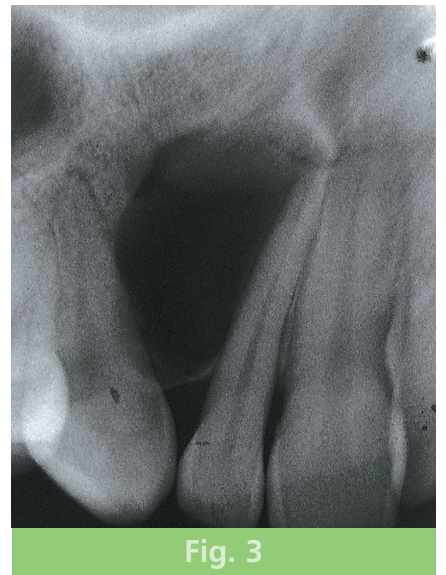

Fig. 3

Fig. 2 et Fig. 3 Radiographies rétro-alvéolaire et orthopantomogramme préopératoire : image radioclaire bien limitée de forme ovalaire siégeant entre la 12 et la 13.

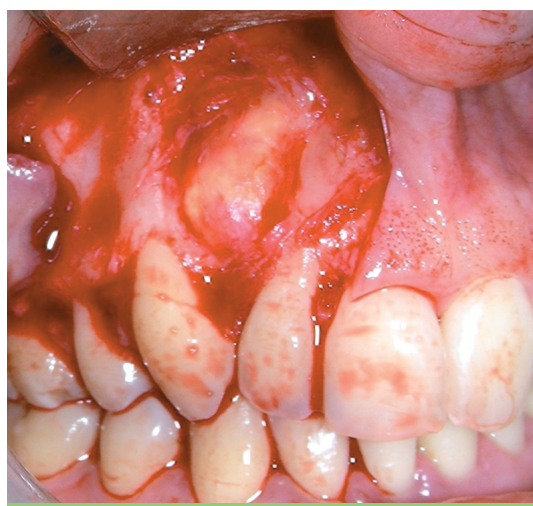

Fig. 4 Vue peropératoire: après décollement mucopériosté, mise en évidence de la lésion.

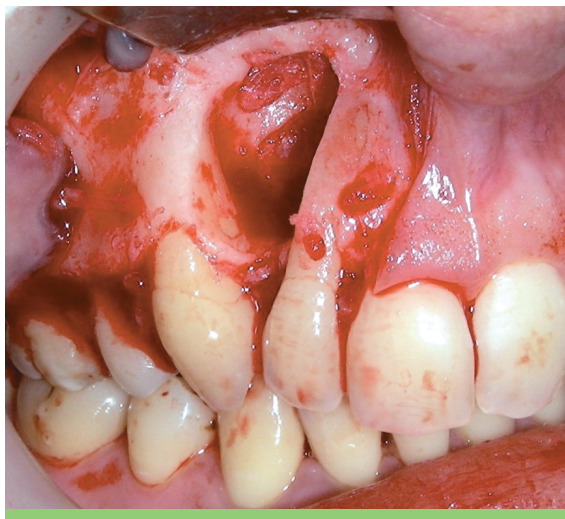

Fig. 5 Vue peropératoire: aspect de la cavité résiduelle après l'énucléation de la lésion.

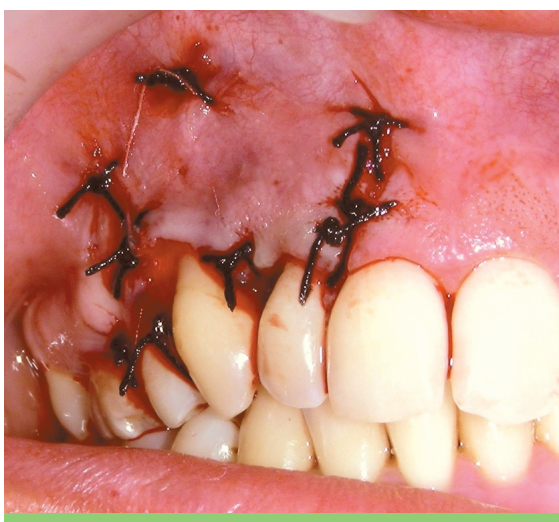

Fig. 6 Vue postopératoire : mise en place des points de sutures.

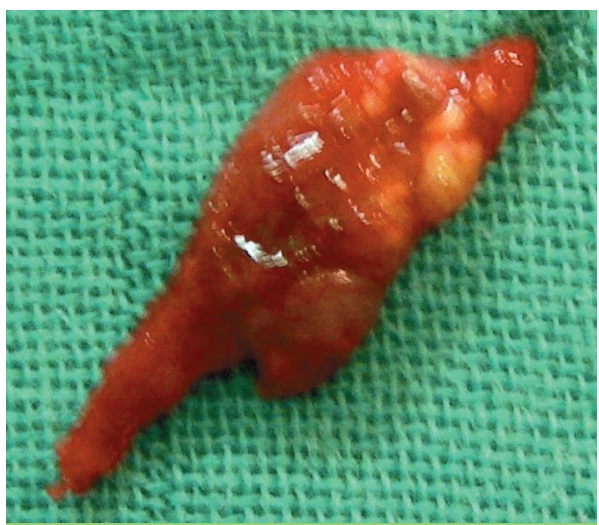

Fig. 7 Pièce opératoire : mesure 2 × 0,5 x $0,2 \mathrm{~cm}$.

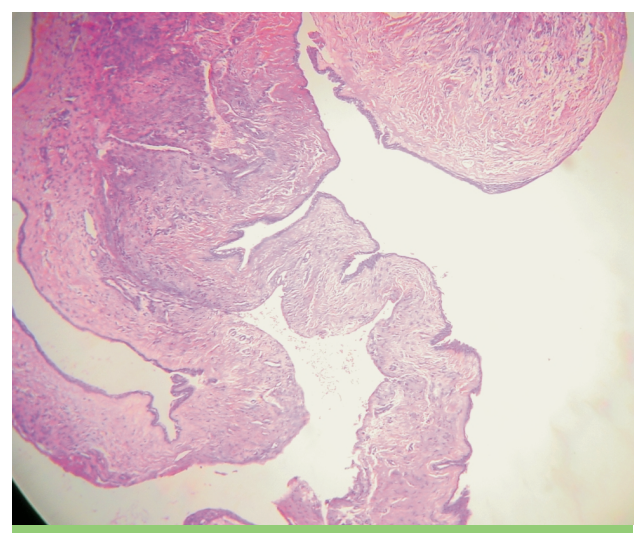

Fig. 8 Coupe histologique: présence d'un épithélium fin présentant des invaginations tapissant un chorion inflammatoire (HE Gx100). 


\section{Discussion}

Épidémiologiquement, le kyste parodontal latéral est d'incidence très faible puisqu'il ne représente que 0,8 à 1,5\% de tous les kystes des maxillaires [1-4].
Ce kyste présente une prédominance au niveau mandibulaire, plus particulièrement au niveau de la région des prémolaires [5-8] (tableau I).

\section{Tableau I}

Siège du kyste parodontal latéral.

\begin{tabular}{|c|c|c|c|}
\hline \multirow{2}{*}{ Auteurs } & \multirow{2}{*}{ Nombre de cas } & \multicolumn{2}{|c|}{ Siège } \\
\hline & & Mandibulaire & Maxillaire \\
\hline \multirow[t]{2}{*}{ Cohen (1984) } & 37 & $29(78,3 \%)$ & $8(21,6 \%)$ \\
\hline & & \multicolumn{2}{|c|}{ Région canine-prémolaire ++ } \\
\hline \multirow[t]{2}{*}{ Rasmusson (1991) } & 32 & $28(88 \%)$ & $4(12 \%)$ \\
\hline & & & Région incisivo-canine ++ \\
\hline \multirow[t]{2}{*}{ Carter (1996) } & 24 & $21(87,5 \%)$ & $3(12,5 \%)$ \\
\hline & & Région prémolaire $(70,8 \%)$ & \\
\hline
\end{tabular}

Dans le cas rapporté, la lésion siégeait au niveau de la région incisivo-canine maxillaire ce qui est peu fréquent.

Le kyste parodontal latéral affecte principale- ment les adultes âgés de 14 à 82 ans, avec absence de prédilection sexuelle $[5-7,9,10]$ (tableau II).

\section{Tableau II}

L'âge et le sexe des patients présentant un kyste parodontal latéral.

\begin{tabular}{lcccccc}
\hline \multirow{2}{*}{ Auteurs } & Nombre & \multicolumn{2}{c}{ Sexe } & Intervalle d'âge & $\begin{array}{c}\text { Âge moyen } \\
\text { (ans) }\end{array}$ \\
\cline { 3 - 4 } & de cas & Femme & Homme & & (ans) \\
\hline Cohen (1984) & 37 & 19 & 18 & $21-82$ & 54 \\
\hline Rasmusson (1991) & 31 & 10 & 22 & $26-77$ & 55 \\
\hline Carter (1996) & 25 & 12 & 13 & $14-78$ & 46 \\
\hline
\end{tabular}

Carter [5] a rapporté une différence statistiquement significative de l'âge moyen des patients en fonction de leur sexe. II a trouvé que l'âge moyen des femmes (40 ans) est de 17 ans de moins par rapport à l'âge moyen des hommes (58 ans). Cela peut suggérer une implication hormonale dans la pathogénie des kystes parodontaux. Cependant, d'autres études sont nécessaires pour explorer le rapport entre le kyste parodontal latéral, la grossesse, la ménopause et la pharmacologie hormonale. 
Sur le plan étiopathogénique, l'histogenèse du kyste parodontal latéral demeure incertaine et plusieurs hypothèses proposées sont en faveur de son origine à partir des vestiges de la lame dentaire [2-4].

Wysocki [10] a noté que la majorité des kystes parodontaux latéraux se développent sur l'os alvéolaire vestibulaire, ce qui correspond à une distribution des vestiges de la lame dentaire, mais pas avec celle des restes de Malassez qui entourent les racines dentaires particulièrement au niveau de la région apicale.

D'après Shear et Pindborg [5], les épaississements épithéliaux en «plaque» caractéristiques du kyste parodontal latéral représentent un exemple de la récapitulation de l'épithélium odontogène dans des conditions pathologiques. Ce processus simule le début du développement dentaire avec un épaississement de l'ectoderme du "stomodeum» (cavité buccale primitive) pour former la lame dentaire. En plus, une activité enzymatique similaire entre les restes de la lame dentaire et l'épithélium du kyste parodontal latéral a été trouvée [7].

Cliniquement, le kyste parodontal latéral est généralement asymptomatique. Parfois, il peut se manifester par une tuméfaction indolore siégeant au niveau vestibulaire. Les dents adjacentes sont respectées et vivantes.

En l'absence de symptomatologie clinique, la découverte du kyste parodontal latéral est fortuite à l'examen radiographique. Dans ce cas, il se présente comme une image radioclaire, unique, bien circonscrite, de forme ronde à ovalaire, de taille typiquement inférieure à $10 \mathrm{~mm}$ et localisée au niveau de l'os interradiculaire [2-7].

Cette image radiologique est non spécifique et doit être différenciée des autres kystes et tumeurs bénignes odontogènes à savoir : le kyste radiculaire latéral, le kyste globulomaxillaire [9], le kyste odontogène résiduel [12] et la tumeur odontogène adénomatoïde (tableau III).

\section{Tableau III}

Diagnostic différentiel radiologique du kyste parodontal latéral.

\begin{tabular}{lll}
\hline \multicolumn{1}{c}{ Lésion } & \multicolumn{1}{c}{ Siège } & \multicolumn{1}{c}{$\begin{array}{c}\text { Vitalité pulpaire des dents } \\
\text { adjacentes }\end{array}$} \\
\hline Kyste parodontal latéral & $\begin{array}{l}\text { Région prémolaire } \\
\text { mandibulaire }\end{array}$ & Vivantes \\
\hline Kyste radiculaire latéral & $\begin{array}{l}\text { En regard d'un canal } \\
\text { endodontique accessoire }\end{array}$ & $\begin{array}{l}\text { Nécrosée ou } \\
\text { traitée endodontique } \\
\text { Fracture radiculaire }\end{array}$ \\
\hline $\begin{array}{l}\text { Kyste globulo-maxillaire } \\
\text { Kyste odontogène résiduel }\end{array}$ & $\begin{array}{l}\text { Entre incisive latérale et } \\
\text { canine maxillaire }\end{array}$ & Vivantes \\
\hline $\begin{array}{l}\text { Tumeur odontogène édentée } \\
\text { adénomatoïde } \\
\text { (stade de début) }\end{array}$ & Région canine maxillaire & Vivantes \\
\hline
\end{tabular}


Le diagnostic final du kyste parodontal latéral est anatomopathologique.

Histologiquement, ce kyste est caractérisé par un épithélium fin (deux à trois couches cellulaires) avec des cellules claires riches en glycogène. Ces cellules apparaissent souvent en «plaque» provoquant une zone d'épaississement épithélial. L'interface, avec le conjonctif sous-jacent, est rectiligne et présente rarement des infiltrats inflammatoires [2-5].

Cet aspect histologique est également trouvé dans le kyste odontogène botryoïde et le kyste gingival de l'adulte (tableau IV).
Le kyste odontogène botryoïde est considéré comme une variété polykystique du kyste parodontal latéral [13-19].

Le kyste gingival de l'adulte présente une localisation gingivale et peut entraîner à la longue une érosion cupuliforme de la corticale osseuse externe ce qui peut le confondre avec le kyste parodontal latéral. Angelopoulou [8] a proposé l'évaluation peropératoire de l'angle formé entre l'os alvéolaire vestibulaire et la cavité kystique pour les différencier. II a montré que, dans le kyste gingival, l'angle formé est obtu, alors que dans le kyste parodontal l'angle formé est aigu.

\section{Tableau IV}

Diagnostic différentiel macroscopique du kyste parodontal latéral.

\begin{tabular}{lll}
\multicolumn{1}{c}{ Lésion } & \multicolumn{1}{c}{ Siège } & \multicolumn{1}{c}{ Aspect } \\
\hline Kyste parodontal latéral & Intra-osseux & Unikystique \\
\hline Kyste gingival de l'adulte & Gingival & Uni- ou polykystique \\
\hline Kyste odontogène botryoïde & Intra-osseux & Polykystique \\
\hline
\end{tabular}

Le traitement du kyste parodontal latéral est chirurgical et consiste en l'énucléation complète de la lésion tout en préservant le desmodonte des dents avoisinantes [2-4]. La vitalité pulpaire des dents adjacentes doit être contrôlée régulièrement.

Cette lésion kystique présente généralement une évolution lente. Rasmusson [7] a rapporté, dans une série de 4 cas de kyste parodontal latéral non traités et suivis pendant 5 à 14 ans, une moyenne de croissance de 0,7 mm/an.
Des cas de récidives rares ont été rapportés dans la littérature ce qui impose une surveillance radiologique et clinique postopératoire pendant plusieurs années $[5,7]$.

Le spectre de transformation néoplasique du kyste parodontal latéral est similaire à celui des autres kystes odontogènes et inclut le développement d'améloblastome unikystique et de carcinome à cellules squameuses $[5,20]$. 


\section{Conclusion}

Le kyste parodontal latéral est une lésion odontogène bénigne de découverte radiologique fortuite à son stade initial. II se présente comme une image radioclaire bien limitée siégeant au niveau de l'os alvéolaire interradiculaire de deux dents voisines clini- quement vitales. L'attitude thérapeutique face à cette lésion consiste en l'énucléation chirurgicale associée à un suivi régulier par des contrôles cliniques de la vitalité pulpaire et radiologique pour surveiller la régénération osseuse.

\section{Bibliographie}

1. Amiri-Jezeh $\mathrm{M}$, Marinello $\mathrm{CP}$, Radics $\mathrm{P}$.

Le kyste parodontal

latéral :

présentation

d'un cas clinique.

Rev Mens Suisse

Odontostomatol

2002;112:1135-1137.

2. Eliasson $\mathrm{S}$, Isacsson $\mathrm{G}$,

Kondell PA.

Lateral periodontal cysts.

Clinical, radiographical and histopathological findings.

Int J Oral Maxillofac Surg 1989;18:191-193.

3. Saygun I, Ozdemir A, Safali M.

Lateral Periodontal Cyst. Turk J Med Sci 2001;31:375-378.

4. Soares de Lima $A$, Machado M, Braga A, Helena de Souza M. Lateral periodontal cyst: aetiology, diagnosis and clinical significance. A review and report of case.

Rev de Clín Pesq Odontol 2005;1:55-59.
5. Carter LC, Carney Y L, Perez-Pudlewski D. Lateral periodontal cyst: Multifactorial analysis of a previously unreported series. Oral Surg Oral Med Oral Pathol Oral Radiol Endod 1996;81:210-216.

6. Cohen D A, Neville BW, Damm DD, White DK.

The lateral periodontal cyst.

A report of 37 cases.

J Periodont

1984;55:230-234.

7. Rasmusson LG,

Magnusson BC, Borrman $\mathrm{H}$.

The lateral

periodontal cyst.

A histopathological and radiographic study of 32 cases.

Brit J Oral. Maxillofac Surg 1991;29(1):54-57.

8. Angelopoulou E., Angelopoulos AP. Lateral periodontal cyst. Review of the litterature and report of a case. J Periodont 1990;61:126-131.
9. Altini $M$, Shear $M$.

The lateral periodontal cyst: an update.

J Oral Pathol Med 1992;21:245-250.

10. Wysocki GP, Brannon RB, Gardner DG, Sapp P. Histogenesis of the lateral periodontal cyst and the gingival cyst of the adult. Oral Surg Oral Med Oral Pathol 1980;50:327-334.

11. Sapp JP, Eversole LR, Wysocki GP. Contemporary oral and maxillofacial pathology (second edition). Mosby édit, Missouri 2004.

12. Mendes RA, Van Der Waal I. An unusual clinicoradiographic presentation of a lateral periodontal cyst report of two cases. Med Oral Patol Oral Cir Bucal 2006;11:185-187.

13. Kaugars GE. Botryoid odontogenic cyst. Oral Surg. Oral Med.

Oral Pathol 1986;62:555-559. 
14. Phelan JA, Kritchman D,

Fusco-Ramer M,

Freedman P D,

Lumerman $\mathrm{H}$.

Recurrent botryoid

odontogenic cyst

(lateral periodontal cyst).

Oral Surg Oral Med

Oral Pathol 1988;66:345-348.

15. Heikinheimo $K$,

Happonen RP, Forssell K,

Kuusilehto A, Virtanen I.

A botryoid

odontogenic cyst

with multiple

recurrences.

Int J Oral Maxillofac Surg

1989;18:10-13.

16. Machado de Sousa SO, Campos AC, Santiago JL, Jaeger RG,

Cavalcanti de Aratijo V.
Botryoid

odontogenic cyst:

report of a case

with clinical

and histogenetic

considerations.

Brit J oral Maxillofac Surg

1990;28:275-276.

17. Redman RS,

Whitestone BW, Winne CE,

Hudec MW, Patterson RH.

Botryoid

odontogenic cyst:

report of a case

with histologic evidence

of multicentric origin.

Int J Oral Maxillofac Surg

1990;19:144-146.

18. Uçok $O$, Yaman $Z$,

Gunhan O, Uçok C,

Dogan N, Baykul T.

Botryoid odontogenic cyst:

report of a case

with extensive

epithelial proliferation.

Int J Oral Maxillofac Surg

2005;34:693-695.

19. Wheathers DR,

Waldron CA.

Unusual multilocular cysts

of the jaws

(botryoid odontogenic

cysts).

Oral Surg 1973:36:235-241.

20. Baker RD, $D^{\prime}$ Onofrio ED,

Corio R, Crawford BE,

Terry BC.

Squamous cell

carcinoma arising

in a lateral periodontal

cyst.

Oral Surg Oral Med

Oral Pathol 1979;47:495-499.

\section{SUMMARY}

\section{Lateral periodontal cyst}

Saliha CHBICHEB,

Abdelkader BENNANI,

Bouchra TALEB,

Wafaa EL WADY

\section{Keywords \\ - lateral periodontal cyst \\ - adult gingival cysts \\ - odontogenic cysts}

The lateral periodontal cyst is classified among odontogenic cysts of the jaws (WHO 1992). The authors report the case of a lateral periodontal cyst found in a 33 year-old white woman. Clinically, it manifested by a swelling sitting in the anterior maxillary region. Radiologically, it presented by a radiolucency sitting between the roots of the 12 and the 13. After initial surgical removal, no recurrence has been reported.

The lateral periodontal cyst presents non specific clinical, radiological and histological signs and creates a differential diagnosis problems with the cysts and the benign odontogenic tumors of the jaws. 


\section{Questions - Réponses}

Réponse par vrai ou faux

1/ Kyste parodontal latéral :

a - Kyste non odontogène des maxillaires

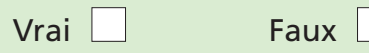

b - Cliniquement asymptomatique et découverte radiologique fortuite

$$
\text { Vrai }
$$$$
\text { Faux }
$$

c - La forme la plus fréquente des kystes des maxillaires

$$
\text { Vrai } \square
$$

Faux

d - Siège électif au niveau région prémolaire mandibulaire

$$
\text { Vrai }
$$

Faux

e - Origine à partir des vestiges de la lame dentaire

$$
\text { Vrai } \square \quad \text { Faux }
$$

f - Siège entre les racines des dents nécrosées

$$
\text { Vrai } \square \quad \text { Faux }
$$

g - Se présente radiologiquement comme une image multiloculaire

$$
\text { Vrai } \square \quad \text { Faux }
$$

h - Le kyste odontogène botryoïde est :

une variété polykystique du kyste parodontal latéral

$$
\text { Vrai } \square \quad \text { Faux }
$$

2/ Le traitement du kyste parodontal latéral :

a - Consiste en l'énucléation chirurgicale

$$
\text { Vrai } \square \quad \text { Faux }
$$

b - Pas de récidive après le traitement

$$
\text { Vrai } \square \quad \text { Faux } \square
$$

c - Présente un risque de transformation néoplasique

$$
\text { Vrai } \square \quad \text { Faux }
$$


